\title{
Mehr Daten? Mehr denken? Umdenken!
}

\author{
Harald Walach \\ Institut für transkulturelle Gesundheitswissenschaften, Kulturwissenschaftliche Fakultät, Europa-Universität Viadrina, Frankfurt/O., \\ Deutschland
}

Ein einleuchtendes, wenn auch naives Narrativ darüber, wie komplementärmedizinische und naturheilkundliche Ansätze in den Mainstream integriert werden können, geht so: Man muss mithilfe der besten vorhandenen Methoden, die von allen akzeptiert sind, empirisch belegen, dass naturheilkundlich-komplementärmedizinische Therapien wirken; dann werden sie auch von der Medizin an den Universitätskliniken anerkannt, von Universitätslehrern gelehrt, in die Versorgung aufgenommen und von Kassen bezahlt. Das bedeutet, randomisierte, wenn möglich Placebo-kontrollierte Studien durchzuführen, die dann am Ende zu Metaanalysen zusammengefasst werden können. Wenn das erreicht ist, bricht das goldene Zeitalter der Integration und der ganzheitlichen Patientenversorgung an. Ich muss gestehen, ich habe selbst lange an dieses Narrativ geglaubt. Und - das wollen will all denen $\mathrm{zu}$ Gute halten, die es immer noch vertreten - es ist ja auch sehr überzeugend. Denn es lehnt sich an eine weitverbreitete Meinung an, wie Wissenschaft funktioniert: über die Anhäufung empirisch valide gesammelter Daten, die irgendwann einmal Sachverhalte als richtig und gültig belegen, sodass man sich darauf verlassen kann und daraus vielleicht eine gültige Theorie entwickelt, die wiederum neue empirische Sachverhalte entdecken lässt. So ähnlich scheinen sich die meisten aktiven Wissenschaftler den Gang der Wissenschaft vorzustellen, auch wenn die Wissenschaftsforschung schon längst gezeigt hat, dass dies allenfalls für Teildomänen, Teilbereiche oder bestimmte Zeiten des wissenschaftlichen Handelns zutrifft [1].

Wenn dieses Narrativ stimmt, so stellt sich die Frage, warum systematische Übersichtsarbeiten, wie die der Arbeitsgruppe um Jost Langhorst zur Naturheilkunde und Komplementärmedizin in den deutschen Leitlinien [2], mit großer Regelmäßigkeit zeigen, dass die Verfahren der Komplementärmedizin unterrepräsentiert sind? Obwohl es in vielen Bereichen gute Daten und nützliche Verfahren gibt, tauchen sie entweder in den Leitlinien nicht auf oder die Studien sind nicht gründlich zur Kenntnis genommen oder werden aus unerfindlichen Gründen anders bewertet als die von konventionellen Behandlungen. Warum tobt um ein Verfahren wie die Homöopathie ein erbitterter Krieg, obwohl gute empirische
Befunde vorliegen, die häufig nicht einmal zur Kenntnis genommen werden [3, 4]? Warum werden Medikamente wie selektive Serotonin-Wiederaufnahmehemmer (SSRIs) zwanglos als wirksam und vernünftig akzeptiert, obwohl allen Kennern der Materie klar ist, dass sie für ganz andere Zwecke entwickelt wurden $[5,6]$ ? Warum wird zur gleichen Zeit Akupunktur bei Rückenschmerzen als eine Methode bezeichnet, bei der man Acht geben müsse, dass sie nicht zu Abhängigkeit führt und sie daher nicht unbedingt zu empfehlen sei [7]?

Ich könnte die Reihe der Merkwürdigkeiten, Fragen und Verwunderungen noch lange fortführen. Aber es reicht vielleicht, um einen simplen Sachverhalt klarzustellen: Das Mainstream-Narrativ vom Primat guter Daten greift zu kurz. Gute Daten sind notwendig, in jedem Fall aber nicht hinreichend als Bedingung für die Akzeptanz und Etablierung einer therapeutischen Methode. Mindestens genauso wichtig ist - neben dem Rahmen der ökonomischpolitischen Machtverhältnisse [8-13] - die Voraussetzung, wie die zu untersuchende Methode in das momentan akzeptierte Theoriegebäude passt; mit anderen Worten: neben den konzeptuellen Hürden gibt es theoretische Barrieren, die der Gleichberechtigung der Komplementärmedizin im Wege stehen. Denn wir bewerten Befunde nie so, wie sie daherkommen - als würde Venus frisch dem Meer entsteigen und wir sie zum ersten Mal bewundern. Wir sehen alle empirischen Daten, egal ob sie aus Studien kommen oder sich unserer gewöhnlichen Wahrnehmung präsentieren, vor dem Hintergrund dessen, was wir erwarten. Und dieser Hintergrund ist vorgeformt und geprägt durch das allgemeine Weltmodell, in dem wir leben und durch konkrete, daraus abgeleitete Erwartungen. Daher würden wir den Ausruf «Schau, es schneit!» im Winter, während einer Woche wiederholter Schneegestöber, anders bewerten als mitten im August. Wenn ein empirischer Befund in das passt, was wir erwarten, sind uns auch wenig spektakuläre, methodisch fragwürdige Daten Beweis genug. Wenn ein empirischer Befund unser Weltmodell und unsere Erwartungen grundlegend durcheinander würfelt, dann würde nicht einmal ein extrem stark abgesichertes experimentelles Ergebnis unser Wohlwollen finden.

\section{KARGER}

Fax +497614520714
(๑) 2016 S. Karger GmbH, Freiburg

$1661-4119 / 16 / 0231-0003 \$ 39.50 / 0$
Prof. Dr. Dr. phil. Harald Walach

Institut für transkulturelle Gesundheitswissenschaften

Kulturwissenschaftliche Fakultät, Europa-Universität Viadrina

Große Scharnstraße 59, 15230 Frankfurt/O., Deutschland

walach@europa-uni.de 
Warum ist das so? Das hängt damit zusammen, dass wir alle Bayesianer sind. Der irische Pastor Bayes hat im 18. Jahrhundert klar gesehen, dass das Potenzial eines empirischen Befundes, die Meinung eines Menschen zu verändern, nicht davon abhängt, wie stark der Befund ist, sondern wie die vorgefasste Meinung dessen ist, der den Befund zu Kenntnis nimmt, die sogenannte «prior probability» oder hier «apriorische Wahrscheinlichkeit» genannt. Ist diese sehr stark in eine bestimmte Richtung vorgeprägt, muss ein Befund in die Gegenrichtung noch viel stärker sein, um die Meinung ändern zu können. Umgekehrt wird ein schwacher Befund in eine bereits vorgegebene Richtung die bereits bestehende Meinung in virtuelle Sicherheit verwandeln. So werden einen Pharmakologen, der von der Gültigkeit der Rezeptor-Liganden-Theorie der pharmakologischen Wirksamkeit als einziger Möglichkeit pharmakologischer Therapie überzeugt ist, auch noch so viele Befunde über die Wirksamkeit der Akupunktur oder der Homöopathie nicht umstimmen können. Umgekehrt wird ihn auch ein schwaches Datenbeispiel von der Möglichkeit der Wirksamkeit eines neuen SRRIs überzeugen, wenn er erst einmal die Serotonin-Mangel-Hypothese der Depression geschluckt hat.

Statistisch-methodisch gesehen verbirgt sich dahinter folgendes: Bayes hat gezeigt, dass die Wahrscheinlichkeit, die sich aus einer empirischen Studie ergibt, die sogenannte posteriore oder empirische Wahrscheinlichkeit, nicht «nackt» gesehen werden kann [14, 15]. Vielmehr muss sie mit der apriorischen oder der vorgängigen Wahrscheinlichkeit verrechnet werden. Erst aus der Interaktion der beiden ergibt sich die finale Wahrscheinlichkeit, dass ein empirischer Sachverhalt von einem Rezipienten akzeptiert wird. Daraus können wir einfach folgern: Ist die apriorische Wahrscheinlichkeit, dass « $\mathrm{X}$ » der Fall ist, bei einem Rezipienten «a» sehr klein, dann muss die posteriore oder empirische Wahrscheinlichkeit «p» für «X» extrem groß sein (oder die Irrtumswahrscheinlichkeit «p» sehr, sehr klein), damit Rezipient «a» seine Meinung zu revidieren bereit ist, und umgekehrt.

Nun wird in der Regel übersehen, dass die statistische Methode, die als Norm definiert wird, d.h. unsere «frequentistische», Fishersche Statistik, ein Sonderfall ist, nämlich für die Situation, in der ein Rezipient eine A-priori-Wahrscheinlichkeit für das Eintreffen eines empirischen Sachverhalts von 0.5 hat. Er findet also, dass das Auftreten von «X» oder das nicht Auftreten von «X» gleich wahrscheinlich ist. Er würde also mit demselben Gleichmut im Sommer wie im Winter aus dem Fenster schauen, wenn einer sagt: «Schau, es schneit!». Er würde die Befunde, dass Akupunktur bei Rückenschmerzen oder Homöopathie bei Husten wirkt mit der gleichen indifferenten Haltung betrachten wie den Befund, dass irgendein monoklonaler Antiköper gegen rheumatoide Arthritis hilft. Und schon jetzt wissen wir: Diese Voraussetzung ist praktisch nie erfüllt und damit die Voraussetzung der Anwendung und konventionellen Interpretation einer solchen Statistik gar nicht gegeben. Denn jede Pharmafirma hat eine Unmenge von Daten erhoben und klare Erwartungen, bevor sie mit einer neuen Substanz in eine Phase3-Studie geht - das gilt auch für die Erwartungen der Forschergemeinde, die in Vorbefunden, Theorien und bestehenden Daten begründet sind, d.h. empirisch erhaltene Daten werden gemäß der Vorerwartung interpretiert. Wer das genauer nachvollziehen will, kann dies mit einem Bayes-Rechner im Internet einmal ausprobieren (z.B. http://graphpad.com/quickcalcs/interpretPValue1/): man stelle sich eine beliebige Ausgangswahrscheinlichkeit vor - hoch, mittel, klein - und gebe ein fiktives Studienergebnis ein. Man kann dann sehen, wie sich die aposteriorische Wahrscheinlichkeit verhält, also die Wahrscheinlichkeit, die sich als Ergebnis eines empirischen Befundes unter Maßgabe der A-priori-Wahrscheinlichkeit ergibt.

Wenn man sich diese Tatsache einmal vor Augen führt, dann sieht man rasch: wenn die A-priori-Wahrscheinlichkeit klein ist, werden auch sehr viele empirische Befunde nichts ausrichten. Denn es kommt ja noch ein psychologischer Faktor hinzu, der in die Bayes-Statistik nur beschränkt eingehen kann: Wir lieben in der Regel unsere Vorurteile, und selbst wenn empirische Befunde ihnen widersprechen, lassen wir unsere Geliebten nicht so einfach ziehen. Man müsste also in eine Bayessche Berechnung noch einen Beharrungsfaktor einkalkulieren, der aber leider nicht quantifizierbar ist, jedoch höchstwahrscheinlich größer, als eine rein wissenschaftlich begründbare A-priori-Wahrscheinlichkeit. Denn wir Menschen sind in der Regel konservativ und denkfaul. Damit sich also das Verhalten von Bundesausschüssen, Leitlinienkommissionen und Menschen mit Entscheidungsmacht im System verändert, braucht es mehr als Daten. Wir benötigen vor allem und flankierend auch «die Arbeit am Begriff», also theoretische Argumentation sowie Dekonstruktion von dogmatischen Denkformen und unreflektierten Annahmen, um die apriorischen Gewichte zu verschieben und die Empirie wirklich wieder zu einem Schiedsrichter machen zu können. Das ist auch der Grund, weswegen es ziemlich naiv ist zu glauben, gute, solide empirische Daten würden reichen. Wenn dem so wäre, dann müssten SSRIs längst aus der Erstattung verbannt sein, Ginkgo und andere wirksame Phytotherapeutika müssten erstattungsfähig sein, Psychotherapie müsste verbreiteter sein als Pharmakotherapie, Akupunktur müsste bei verschiedenen Schmerzsyndromen als Methode der ersten Wahl gelten und NSAIDs aufgrund des mangelnden Wirkungsprofils und der ungünstigen Nebenwirkungen auf die hinteren Ränge gewechselt sein (wie das übrigens in England der Fall ist [16]).

Die Tatsache, dass das bei uns nicht so ist, zeigt uns: Empirie allein ist schön, aber nicht ausreichend. Wir müssen auch - oder vielleicht: vor allem? - die dogmatischen Strukturen reflektieren und diskutieren, die zur Fixierung der Annahmenstruktur führen, unter der empirische Ergebnisse betrachtet und verwertet werden; und wir müssen uns darüber im Klaren sein: vor allem diese Annahmestruktur ist es, die einer Integration von Komplementärmedizin und Naturheilverfahren im Weg steht, nicht die empirischen Ergebnisse. Von diesen könnten wir zwar durchaus mehr haben, aber das gilt immer und für alle Gebiete. Wir brauchen nicht mehr Daten, sondern mehr, und vor allem anderes Denken. Und das bedeutet: Umdenken, was das Verhältnis von Daten und Theorie angeht. 


\section{Literatur}

1 Walach H: Psychologie: Wissenschaftstheorie, philosophische Grundlagen und Geschichte, ed 3. Stuttgart, Kohlhammer, 2013.

2 Klose P, Kraft K, Cramer H, Lauche R, Dobos G, Langhorst J: Phytotherapie in den medizinischen S3-Leitlinien der Arbeitsgemeinschaft der Wissenschaftlichen Medizinischen Fachgesellschaften - eine systematische Übersichtsarbeit. Forsch Komplementmed 2014;21:388-400.

3 Mathie RT, Lloyd SM, Legg LA, Clausen J, Moss S, Davidson JR, Ford I: Randomised placebo-controlled trials of individualised homeopathic treatment: sytematic review and meta-analysis. Syst Rev 2014;3:142.

4 Walach $\mathrm{H}$, Teut M: Scientific proving of ultra high dilutions in humans. Homeopathy 2015;104:322-327.

5 Healy D: Serotonin and depression. BMJ 2015;350:h1771.

6 Gøtzsche PC: Deadly Medicines and Organised Crime: How Big Pharma Has Corrupted Healthcare. London, Radcliff, 2013.

7 Bundesärztekammer (BÄK), Kassenärztliche Bundesvereinigung (KBV), Arbeitsgemeinschaft der Wissenschaftlichen Medizinischen Fachgesellschaften (AWMF): Nationale Versorgungsleitlinie (NVL) Kreuzschmerz - Langfassung. Version 4.2010. www. leitlinien.de/nvl/kreuzschmerz/ (Zugriff 1.2.16).

8 Brix Bindslev JB, Schroll J, Gøtzsche PC, Lundh A: Underreporting of conflicts of inter est in clinical practice guidelines: cross-sectional study. BMC Med Ethics 2013;14:19.

9 Ransohoff DF, Pignone M, Sox HC: How to decide whether a clinical practice guideline is trustworthy. JAMA 2013;309:139-140.

10 Stamatakis E, Weiler R, Ioannidis JPA: Undue industry influences that distort healthcare research, strategy, expenditure and practice: a review. Eur J Clin Invest 2013;43 469-475.

11 Schott G, Dünnweber C, Mühlbauer B, Niebling W, Pachl H, Ludwig W-D: Does the pharmaceutical industry influence guidelines?: two examples from Germany. Dtsch Arztebl Int 2013;110:575-583.

12 Norris SL, Holmer HK, Ogden LA, Selph SS, Fu R: Conflict of interest disclosures for clinical practice guidelines in the national guideline clearinghouse. PLoS One 2012, 7:e47343.

13 Matheson A: How industry uses the ICMJE guidelines to manipulate authorship - and how they should be revised. PLoS Med 2011;8:e1001072.

14 Mazur DJ: A history of evidence in medical decisions: from the diagnostic sign to Bayesian inference. Med Decis Making 2012;32:227-231.

15 Aickin M: Bayes without priors. J Clin Epidemiol 2004;57:4-13.

16 Savigny P, Kuntze S, Watson P, Underwood M, Ritchie G, Cotterell M, Hill D, Browne N, Buchanan E, Coffey P, Dixon P, Drummond C, Flanagan M, Greenough C, Griffiths M, Halliday-Bell J, Hettinga D, Vogel S, Walsh D: Low back pain: early management of persistent non-specific low back pain. National Collaborating Centre for Primary Care and Royal College of General Practitioners, 2009. 